\title{
Droplet formation in microfluidic cross-junctions
}

\author{
Haihu Liu ${ }^{\text {a) }}$ and Yonghao Zhang ${ }^{\text {b) }}$ \\ Department of Mechanical Engineering, University of Strathclyde, Glasgow G1 1XJ, United Kingdom
}

(Received 11 January 2011; accepted 29 June 2011; published online 4 August 2011)

\begin{abstract}
Using a lattice Boltzmann multiphase model, three-dimensional numerical simulations have been performed to understand droplet formation in microfluidic cross-junctions at low capillary numbers. Flow regimes, consequence of interaction between two immiscible fluids, are found to be dependent on the capillary number and flow rates of the continuous and dispersed phases. A regime map is created to describe the transition from droplets formation at a cross-junction (DCJ), downstream of cross-junction to stable parallel flows. The influence of flow rate ratio, capillary number, and channel geometry is then systematically studied in the squeezing-pressure-dominated DCJ regime. The plug length is found to exhibit a linear dependence on the flow rate ratio and obey power-law behavior on the capillary number. The channel geometry plays an important role in droplet breakup process. A scaling model is proposed to predict the plug length in the DCJ regime with the fitting constants depending on the geometrical parameters. (C) 2011 American Institute of Physics. [doi:10.1063/1.3615643]
\end{abstract}

\section{INTRODUCTION}

Rapid development of microfabrication technologies has facilitated a broad range of microfluidic applications especially in life sciences. Microdroplet technology has recently emerged as a promising flexible platform for microfluidic functions. $^{1-4}$ The miniaturization of entire process enables the rapid analysis of very small quantities of samples in a portable, automated, and inexpensive format. ${ }^{3}$ Recently, microdroplet technology has been used as microreactors for chemical analysis and protein crystallization, ${ }^{5,6}$ as molds for curing polymeric microspheres. ${ }^{7,8}$ Furthermore, programmable fluidic assays for sampling glucose concentration of human physiological fluids ${ }^{9}$ and DNA analysis ${ }^{10}$ have been individually demonstrated using microdroplet system. As samples/reagents are confined in droplets so that sample dilution caused by Taylor dispersion ${ }^{11}$ can be avoided, and mixing performance can be improved. ${ }^{12}$ In addition, it can avoid sample/surface interaction and thus eliminate surface adsorption and cross sample contamination.

Many microfluidic devices have been designed to generate uniform droplets, including geometry-dominated devices, ${ }^{13,14}$ flow-focusing devices, ${ }^{15-19}$ T-junctions, ${ }^{20-26}$ and co-flowing devices. ${ }^{27,28}$ However, the underlying mechanisms of droplet formation in microchannels have not been well understood, which hinders device optimization and operation. The twophase flow characteristics in microchannels are determined by flow conditions, channel geometry, and fluids properties. Guillot and Colin ${ }^{22}$ experimentally observed that, for a given flow rate of the continuous phase, the flow pattern changes from droplets at $\mathrm{T}$-junction to droplets in channel if the flow rate of the dispersed phase increases. This indicates that for a given capillary number $C a=\eta_{c} u_{c} / \sigma\left(\eta_{c}, u_{c}\right.$ are the viscosity and velocity of the continuous phase; $\sigma$ is the interfacial tension

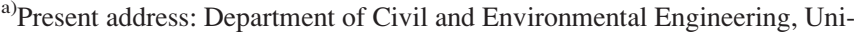
versity of Illinois at Urbana-Champaign, Illinois 61801, USA. Electronic mail: haihuliu@illinois.edu.

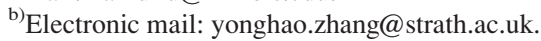

coefficient), when the flow rate ratio $Q=Q_{d} / Q_{c}\left(Q_{d}\right.$ and $Q_{c}$ are volume flow rate of the dispersed and continuous phases, respectively) increases, a flow regime change occurs. With a further increase in $Q$, the flow regime changes to parallel flow. Tan et al. ${ }^{18}$ also found that the two-phase flow patterns depend on the flow rates of the continuous phase and the dispersed phase for the plug formation in a microfluidic cross-junction, which is similar to the observation of Guillot and Colin. ${ }^{22}$

The channel geometry has been found to play an important role in the droplet formation process. For example, Garstecki et al. ${ }^{23}$ identified a squeezing mechanism due to confined microchannel geometry in droplet formation process, which does not exist in an unbounded flow condition. By studying the underlying mechanisms that control the droplet breakup, some scaling laws have been established to predict the size of droplets produced in microfluidic devices. ${ }^{29,30}$ However, the currently available experimental data are still sporadic. Various materials are used to fabricate the microchannels with diverse dimensions, and the experiments have been performed at different flow conditions with different fluids. Consequently, the information is fragmented, which leads to inconclusive and even incompatible findings. Based on statistical analysis of the broad range of available literature data, Steegmans et al. ${ }^{30}$ have shown that none of the scaling models, which are developed to predict droplet formation in a microfluidic T-junction, is general enough to describe the original data and data from other literature sources. Also, they found that the available literature data could be better represented by a two-step model consisting of a growth phase and a detachment phase.

Meanwhile, experiments at such small scales are still difficult. For example, it is challenging to accurately measure local flow fields, droplet deformation, breakup, and coalescence. Numerical study can be complementary to experimental investigation. For example, Menech et al. ${ }^{25}$ numerically identified three distinct mechanisms, i.e., squeezing, dripping, and jetting in droplet formation in a T-junction. However, much effort is still required to numerically simulate 
droplet generation, transportation, and interaction with surface. While the front tracking methods are not suitable for droplet breakup and coalescence, the interface capturing methods such as volume-of-fluid and level set methods will experience numerical instability when interfacial tension becomes a dominant factor in microdroplet behavior. ${ }^{31}$ Recently developed lattice Boltzmann (LB) method has shown great potential for modeling interfacial interactions while incorporating fluid flow as a system feature. ${ }^{32}$ It is a pseudo-molecular method tracking evolution of the distribution function of an assembly of molecules and built upon microscopic models and mesoscopic kinetic equations. ${ }^{33}$ Its mesoscopic nature can provide many advantages of molecular dynamics, making the LB method especially useful for simulation of droplet dynamics. ${ }^{23,34-37}$

In this work, a multiphase lattice Boltzmann model is employed to investigate droplet formation in microfluidic cross-junctions. We will first present our simulation results that reveal the influence of capillary number and flow rate ratio on the flow regime transition from droplet generation at cross-junction (DCJ), downstream of the cross-junction (DC), to parallel flows $(\mathrm{PF})$. In the DCJ regime, the influences of capillary number, flow rate ratio, and channel geometry will be studied in details. We will compare our simulation with the existing models and experimental data. To our best knowledge, this is for the first time that numerical simulations are performed to identify the flow regimes and investigate the effect of channel geometry on the droplet formation in micro- fluidic cross-junctions. This study can provide useful information for understanding microdroplet dynamics and optimal design of multiphase microfluidic devices.

\section{NUMERICAL METHOD}

In the lattice Boltzmann method, a fluid is modeled as pseudo particles, whose distribution function $f_{i}$ is governed by the lattice Boltzmann equation, e.g., using the BhatnagarGross-Krook (BGK) collision operator ${ }^{32}$

$$
f_{i}\left(\mathbf{x}+\mathbf{e}_{i} \delta_{t}, t+\delta_{t}\right)=f_{i}(\mathbf{x}, t)-\frac{1}{\tau}\left[f_{i}(\mathbf{x}, t)-f_{i}^{e q}(\mathbf{x}, t)\right],
$$

where $f_{i}(\mathbf{x}, t)$ is the particle distribution function in the $i$ th velocity direction at the position $\mathbf{x}$ and the time $t, \mathbf{e}_{i}$ is the lattice velocity in the $i$ th direction, $\tau$ is the dimensionless relaxation time, and $f_{i}^{e q}$ is the equilibrium distribution function as a function of local density $\rho$ and velocity $\mathbf{u}$

$$
f_{i}^{e q}=w_{i} \rho\left[1+\frac{\mathbf{e}_{i} \cdot \mathbf{u}}{c_{s}^{2}}+\frac{\left(\mathbf{e}_{i} \cdot \mathbf{u}\right)^{2}}{2 c_{s}^{4}}-\frac{\mathbf{u} \cdot \mathbf{u}}{2 c_{s}^{2}}\right],
$$

where $c_{s}$ denotes the speed of sound which is given by $c / \sqrt{3}$ with $c=\delta_{x} / \delta_{t}$ being the lattice speed and $\delta_{x}$ being the lattice length.

For the three-dimensional 19-velocity (D3Q19) model, the lattice velocity $\mathbf{e}_{i}$ and the weight coefficients $w_{i}$ are given as

$$
\mathbf{e}_{i}= \begin{cases}(0,0,0), & i=0 \\ ( \pm 1,0,0) c,(0, \pm 1,0) c,(0,0, \pm 1) c, & i=1,2, \ldots, 6 \\ ( \pm 1, \pm, 0) c,( \pm 1,0, \pm 1) c,(0, \pm 1, \pm 1) c, & i=7,8, \ldots, 18\end{cases}
$$

$$
w_{i}= \begin{cases}1 / 3, & i=0 \\ 1 / 18, & i=1,2, \ldots, 6 \\ 1 / 36, & i=7,8, \ldots, 18 .\end{cases}
$$

The macroscopic properties including the local density and momentum are related to the particle distribution function $f_{i}$ by

$$
\rho=\sum_{i} f_{i}=\sum_{i} f_{i}^{e q} ; \quad \rho \mathbf{u}=\sum_{i} f_{i} \mathbf{e}_{i}=\sum_{i} f_{i}^{e q} \mathbf{e}_{i} .
$$

Using the Chapman-Enskog expansion for the D3Q19 model, Eq. (1) can lead to the Navier-Stokes equations in the long-wavelength and low-frequency limit ${ }^{38}$

$$
\begin{gathered}
\partial_{t} \rho+\nabla \cdot(\rho \mathbf{u})=0, \\
\partial_{t}(\rho \mathbf{u})+\nabla \cdot(\rho \mathbf{u u})=-\nabla p+\nabla \cdot(\rho \nu \nabla \mathbf{u}),
\end{gathered}
$$

where the pressure and the kinematic viscosity are given as $p=\rho c_{s}^{2}$ and $\nu=(\tau-1 / 2) c_{s}^{2} \delta_{t}$.

Currently, the most applied LB multiphase methods are the color-fluid model ${ }^{39}$ the pseudo-potential model, ${ }^{40}$ and the free-energy model. ${ }^{41,42}$ In the color-fluid model, the procedure of redistribution of the colored density at each node to separate different phases requires time-consuming calculation of local maxima, and the perturbation step with the redistribution of colored distribution functions causes an anisotropic interfacial tension that induces high spurious velocities near interface. ${ }^{38}$ The pseudo-potential model introduces the nearest-neighboring interaction between fluid particles to describe the intermolecular potential, and the phase separation occurs with a properly chosen potential function. Although significant advances have recently been made, ${ }^{43-45}$ further improvements are necessary for the pseudo-potential model to minimize spurious velocities at interface and control numerical instability for the flows with low capillary number and viscosity ratio. The free-energy model suffers from the lack of Galilean invariance, ${ }^{32}$ although the local conservation of mass and momentum is satisfied. Based on the original color-fluid model of Gunstensen et al., the recent improvements have been made by Lishchuk et al. ${ }^{46}$ and Latva-Kokko and Rothman, ${ }^{47}$ which facilitate simulational access to flow regimes of low Reynolds and capillary numbers. In this study, we will use the improved color-fluid model, because the Reynolds number and the capillary number are typically small in microfluidic droplet formation.

In the original color-fluid model of Gunstensen et al., ${ }^{39}$ "Red" and "Blue" particle distribution functions $f_{i}^{R}$ and $f_{i}^{B}$ 
were introduced to represent two different fluids. The total particle distribution function is defined as $f_{i}=f_{i}^{R}+f_{i}^{B}$. Each of the colored phases undergoes the collision and streaming operations

$$
f_{i}^{k}\left(\mathbf{x}+\mathbf{e}_{i}, t+1\right)=f_{i}^{k}(\mathbf{x}, t)-\frac{f_{i}^{k}(\mathbf{x}, t)-f_{i}^{k, e q}(\mathbf{x}, t)}{\tau_{k}}+\Omega_{i}^{k},
$$

where $k=R$ or $B$ denotes the color ("Red" or "Blue"). The viscosity of each fluid can be selected by choosing the desired $\tau_{k}$, i.e., $\nu_{k}=c_{s}^{2}\left(\tau_{k}-1 / 2\right)$. Conservation of mass for each phase and total momentum conservation require

$$
\begin{gathered}
\rho_{k}=\sum_{i} f_{i}^{k}=\sum_{i} f_{i}^{k, e q}, \\
\rho \mathbf{u}=\sum_{i} \sum_{k} f_{i}^{k} \mathbf{e}_{i}=\sum_{i} \sum_{k} f_{i}^{k, e q} \mathbf{e}_{i} .
\end{gathered}
$$

The additional collision operator $\Omega_{i}^{k}$ (perturbation step) contributes to the mixed interfacial regions and generates an interfacial tension

$$
\Omega_{i}^{k}=A_{k}|\mathbf{G}| \cos 2\left(\theta_{i}-\theta_{f}\right),
$$

where $A_{k}$ is a free parameter controlling the interfacial tension, $\theta_{i}$ is the polar angle of the lattice vector $\mathbf{e}_{i}$, and $\theta_{f}$ is the polar angle of the local color gradient $\mathbf{G}$, which is defined as

$$
\mathbf{G}(\mathbf{x}, t)=\sum_{i} w_{i} \mathbf{e}_{i}\left[\rho_{R}\left(\mathbf{x}+\mathbf{e}_{i}, t\right)-\rho_{B}\left(\mathbf{x}+\mathbf{e}_{i}, t\right)\right] .
$$

To promote phase segregation and maintain interface, the socalled recoloring step is applied, which enables to keep the interface sharp, and at the same time prevents the two fluids from mixing with each other. The colors are demixed by maximizing the work done against the color flux $\mathbf{q}(\mathbf{x}, t)$, which is defined by

$$
\mathbf{q}(\mathbf{x}, t)=\sum_{i} w_{i} \mathbf{e}_{i}\left[f_{i}^{R}(\mathbf{x}, t)-f_{i}^{B}(\mathbf{x}, t)\right] .
$$

The perturbation step can introduce anisotropy and high spurious velocities at the interface. Additionally, when applied to study creeping flows, the recoloring step can produce lattice pinning, ${ }^{47}$ a phenomenon that the interface can be pinned or attached to the simulation lattice rendering an effective loss of Galilean invariance. It was also demonstrated that there is an increasing tendency for lattice pinning as both of the capillary and Reynolds numbers decrease. ${ }^{48}$

Lishchuk et al..$^{46}$ used the concept of a continuum surface force $(\mathrm{CSF})^{49}$ to model the interfacial tension. In their algorithm, the perturbation step in the original Gunstensen model was replaced by a direct forcing term at the mixed region. In order to satisfy the stress boundary condition and the continuity of velocity, a local pressure gradient is forced throughout the interface as an additional body force $\mathbf{F}(\mathbf{x}, t)$, which is incorporated into the LB equation by the forcing term $\phi_{i}(\mathbf{x}, t)$ at the collision step. It has been reported that this algorithm can greatly reduce the spurious currents and improve the isotropy of the interface. The body force is defined to act normal to the interface with a magnitude proportional to the gradient of $\rho^{N}$, which is given by

$$
\mathbf{F}(\mathbf{x}, t)=-\frac{1}{2} \sigma \kappa \nabla \rho^{N},
$$

where $\sigma$ is an interfacial tension parameter, $\rho^{N}$ is the phase field defined as

$$
\rho^{N}(\mathbf{x}, t)=\frac{\rho_{R}(\mathbf{x}, t)-\rho_{B}(\mathbf{x}, t)}{\rho_{R}(\mathbf{x}, t)+\rho_{B}(\mathbf{x}, t)}, \quad-1 \leq \rho^{N} \leq 1,
$$

and $\kappa$ is the local interface curvature, which is calculated by

$$
\kappa=-\nabla_{s} \cdot \mathbf{n},
$$

where $\nabla_{s}=(\mathbf{I}-\mathbf{n} \otimes \mathbf{n}) \cdot \nabla$ is the interface gradient operator, and $\mathbf{I}$ is the second-order identity tensor. The unit normal vector $\mathbf{n}$ is defined as a function of phase field

$$
\mathbf{n}=-\frac{\nabla \rho^{N}}{\left|\nabla \rho^{N}\right|} .
$$

Based on the body force term given by Eq. (14), the forcing term $\phi_{i}$ can realize the interfacial tension effect, which is given as ${ }^{46}$

$$
\phi_{i}(\mathbf{x}, \mathbf{t})=\frac{1}{c_{s}^{2}} w_{i} \mathbf{e}_{i} \cdot \mathbf{F}(\mathbf{x}, \mathbf{t}) \delta_{t} .
$$

The calculation of partial derivatives is required to evaluate the interface curvature and the normal vector. To minimize the discretization error, these derivatives are calculated using 19-point finite difference stencils

$$
\frac{\partial \psi(\mathbf{x})}{\partial x_{\alpha}}=\frac{1}{c_{s}^{2}} \sum_{i} w_{i} \psi\left(\mathbf{x}+\mathbf{e}_{i}\right) e_{i \alpha} .
$$

In addition, the original recoloring step is modified by an anti-diffusion scheme proposed by Latva-Kokko and Rothman, ${ }^{47}$ which can solve the lattice pinning problem and creates a symmetric distribution of particles around the interface so that the spurious velocities can be further reduced. Following their method, the post-collision, postsegregation (recolored) particle distribution functions for the red and blue fluids are calculated by

$$
\begin{aligned}
f_{i}^{R} & =\frac{\rho_{R}}{\rho_{R}+\rho_{B}} f_{i}+\beta \frac{\rho_{R} \rho_{B}}{\rho_{R}+\rho_{B}} w_{i} \cos \varphi\left|\mathbf{e}_{i}\right|, \\
f_{i}^{B} & =\frac{\rho_{B}}{\rho_{R}+\rho_{B}} f_{i}-\beta \frac{\rho_{R} \rho_{B}}{\rho_{R}+\rho_{B}} w_{i} \cos \varphi\left|\mathbf{e}_{i}\right|,
\end{aligned}
$$

where $f_{i}$ denotes the post-collision, presegregation value of the total particle distribution function along the $i$ th lattice direction; $\beta$ is the segregation parameter and is fixed at 0.7 to maintain a narrow interface thickness and reduce spurious velocities; ${ }^{50}$ and also, we will show that this choice is necessary to reproduce correct dynamical behavior of droplets; $\varphi$ is the angle between the color gradient $\nabla \rho^{N}$ and the lattice vector $\mathbf{e}_{i}$, which is defined by

$$
\cos \varphi=\frac{\mathbf{e}_{i} \cdot \nabla \rho^{N}}{\left|\mathbf{e}_{i}\right|\left|\nabla \rho^{N}\right|} .
$$

\section{RESULTS AND DISCUSSION}

First, Taylor deformation is used to examine the effect of segregation parameter $\beta$ on droplet dynamical behavior. A 
droplet is placed between two parallel plates that are moving in the opposite directions to obtain a linear shear in the Stokes flow regime (i.e., small Reynolds number). Droplet deformation is studied as a function of the shear rate via the capillary number. The definitions of the Reynolds number and capillary number are given as

$$
R e=\frac{\gamma R^{2} \rho}{\eta}, \quad C a=\frac{\gamma R \eta}{\sigma},
$$

where $\gamma=2 U / H$ is the shear rate with $U$ being the velocity of the moving plate, and $H$ being the channel height; $R$ is the initial radius of the droplet. For this case, we assume that both fluids have the same density and viscosity. The simulations are run at $R e=0.1$ for a droplet with the radius of 10 lattice cells in a system of $100 \times 50 \times 50$ lattice cells. At the steady state, the droplet is assumed to be ellipsoidal, which is usually characterized by the deformation parameter $D f$, defined as

$$
D f=\frac{a-b}{a+b},
$$

where $a$ and $b$ are the lengths of the major and minor axis of the deformed droplet, respectively. For a droplet in the Stokes regime with a low $C a, D f$ follows the Taylor relation as $^{51}$

$$
D f=(35 / 32) C a \text {. }
$$

A series of numerical simulations are performed with $\beta=\{0.5,0.7,1\}$ and $C a$ varying from 0.05 to 0.3 . Fig. 1 shows the time evolution of Taylor deformation parameter for different $C a$ and $\beta$. We can observe that the droplet cannot evolve to a steady state for $\beta=1$, while a steady droplet deformation can be reached for both $\beta=0.5$ and 0.7 . However, a small $\beta$ (i.e., a large interface thickness) usually produces a large droplet deformation at a fixed $\mathrm{Ca}$, which can

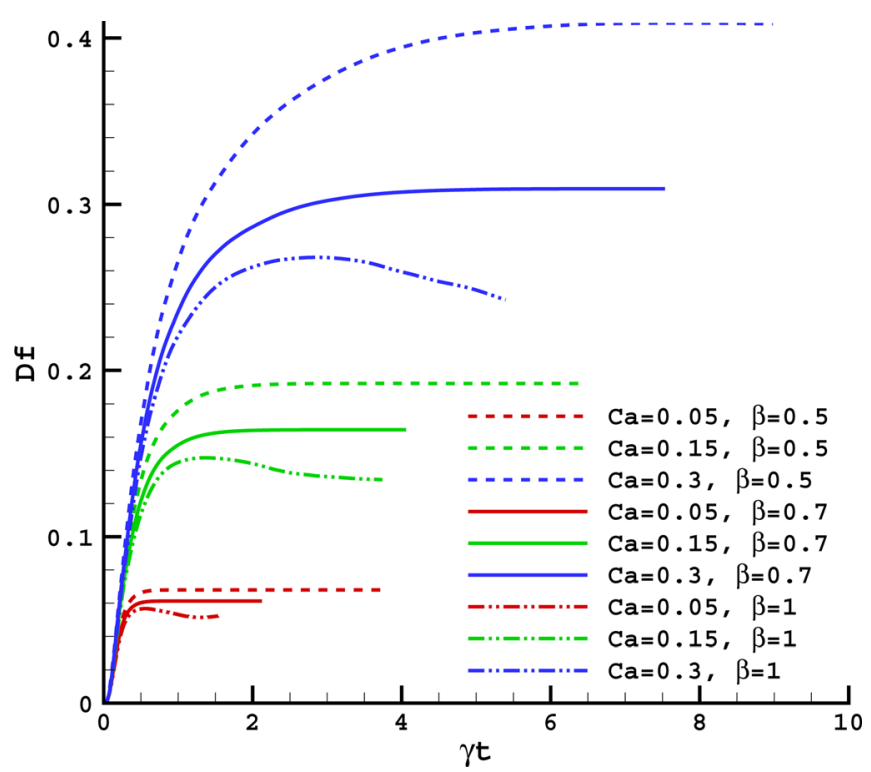

FIG. 1. (Color online) Evolution of the Taylor deformation parameter for $\beta=\{0.5,0.7,1\}$ at $C a=\{0.05,0.15,0.3\}$ and $R e=0.1$.

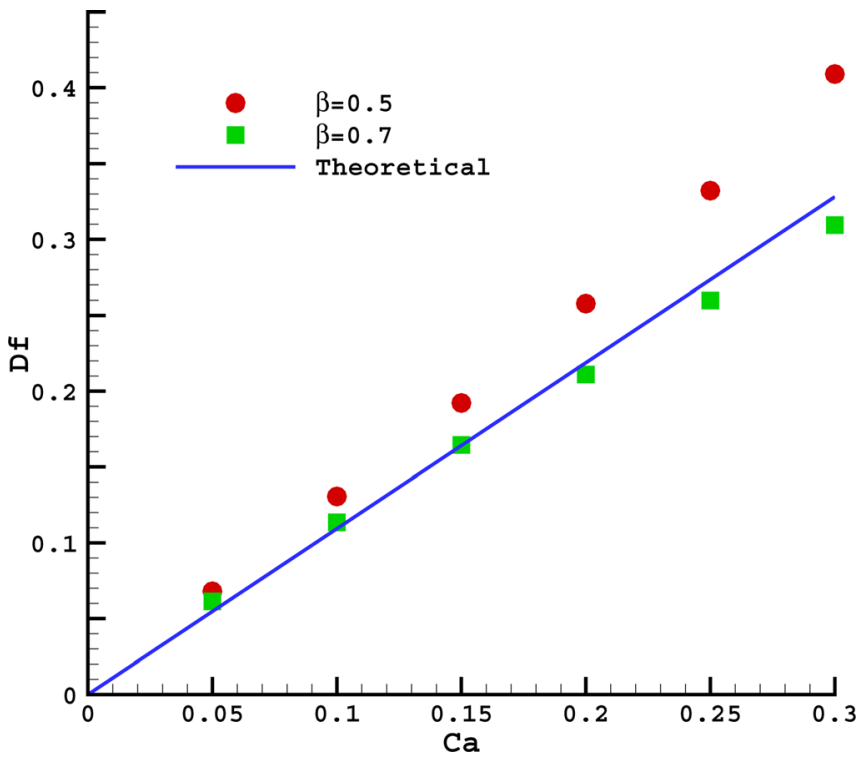

FIG. 2. (Color online) Taylor deformation parameter $D f$ as a function of the capillary number at $R e=0.1$. The solid line is the theoretical Taylor relation given by Eq. (25).

be clearly seen in Fig. 2. Meanwhile, the LB simulations with $\beta=0.7$ are in good agreement with the theoretical Taylor relation. Therefore, we will use $\beta=0.7$ in the following simulations in order to reproduce the correct droplet dynamics.

The geometry of cross-junction microchannel is illustrated in Fig. 3. The microchannel consists of a main channel with width $w_{d}$ and two lateral channels with the same width $w_{c}$. The depth $h$ is uniform throughout the channels. The dispersed phase water is introduced at the inlet of the main channel, and the continuous phase oil is injected into the lateral channels. Halfway bounce-back is used at the solid walls in order to obtain no-slip boundary condition. ${ }^{32}$ We assume that the fluids are pure single-phase at the inlets and outlet, and the constant inlet flow rates and outlet pressure boundary conditions are imposed following Zou and He. ${ }^{52}$ For simplicity, we set the densities of both fluids equal, as the

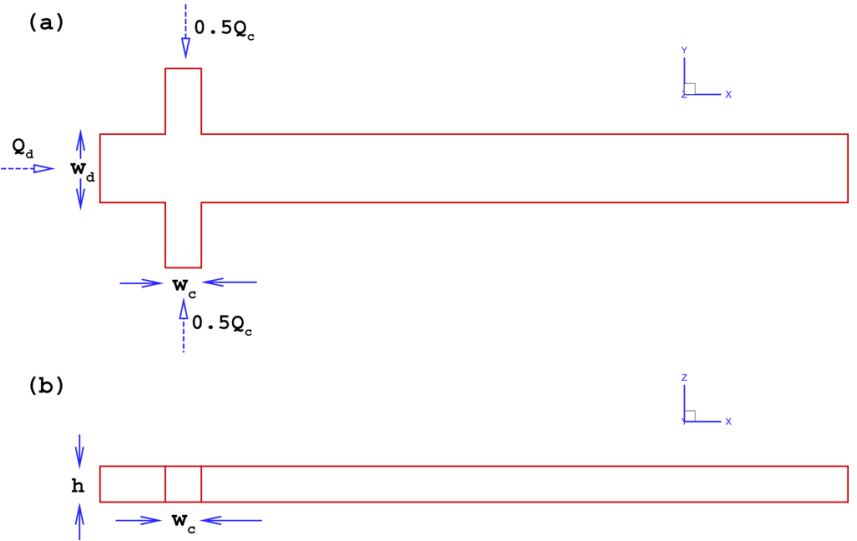

FIG. 3. (Color online) (a) The top and (b) side view of a microfluidic crossjunction, where $w_{d}, w_{c}$ are the width of the main and lateral channels, $h$ is the channel depth, and $Q_{c}$ and $Q_{d}$ are the inlet volumetric flow rates of the continuous and dispersed phases. 


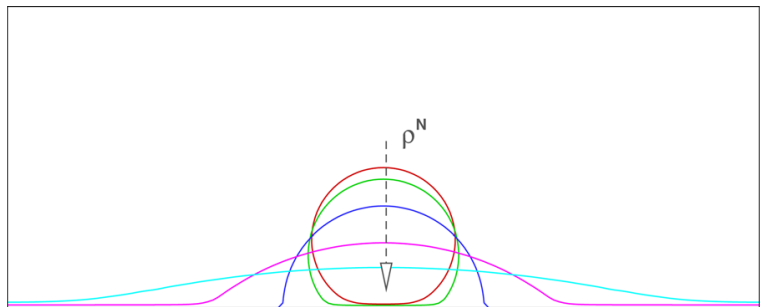

FIG. 4. (Color online) The different contact angles obtained through adjusting the phase field $\rho_{S}^{N}$ at the solid wall. The values of $\rho_{S}^{N}$ are taken as $\rho_{S}^{N}=\{-0.75,-0.5,0,0.5,0.75\}$ along the direction of arrow.

buoyancy-driven velocities in a typical oil-water microfluidic system are negligible. ${ }^{25}$

The wettability of the microchannel walls can strongly influence droplet formation, it is therefore essential to account for the fluid-surface interactions. We follow the assumption made by Rowlinson and Widom ${ }^{53}$ that the solid wall is a mixture of two fluids, thus having a certain value of the phase field $\rho_{S}^{N}$. The derivatives of phase field near the wall boundary can therefore be calculated using Eq. (19). Consequently, the interfacial force term in Eq. (14) becomes dependent on the properties of the neighboring solid lattice sites, resulting in a special case of the wetting boundary condition. Fig. 4 demonstrates that the assigned value of phase field at the solid wall can be used to modify the static contact angle of the interface at the solid surface. Approaches similar to the one described above were also adopted by Bekri and Adler ${ }^{54}$ for the original model of Gunstensen et al. ${ }^{39}$ by van der Graaf $e t$ al. ${ }^{24}$ and Liu and Zhang ${ }^{37}$ in the free-energy models and by Shan and $\mathrm{Chen}^{40}$ in the pseudo-potential model through the fluid-solid interaction potential. In addition, in the free-energy models, the interaction between fluids and solid surface can be modeled by a surface integral that appears in the boundary condition of the free energy. ${ }^{55,56}$ Unless otherwise stated, we assume $\rho_{S}^{N}=-1$ in the following simulations so that the continuous phase completely wets the wall surfaces while the dispersed phase is non-wetting.

The dynamical response of fluids in a microfluidic crossjunction can be described successfully by six independent dimensionless numbers, which are commonly defined by the geometrical and physical parameters including the inlet widths $\left(w_{c}\right.$ and $\left.w_{d}\right)$, the channel depth $(h)$, the interfacial tension $(\sigma)$, the inlet volumetric flow rates $\left(Q_{c}\right.$ and $\left.Q_{d}\right)$, the viscosities $\left(\eta_{c}\right.$ and $\eta_{d}$ ) of the two fluids and their densities $(\rho)$, where the subscripts "c" and "d" denote the continuous and dispersed phases, respectively. The capillary number $(\mathrm{Ca})$, which describes relative importance of the viscous force and the interfacial tension, is the most important parameter for droplet formation. Here, it is defined by the average inlet velocity $u_{c}$ and the viscosity $\eta_{c}$ of the continuous phase and the interfacial tension $\sigma$

$$
C a=\frac{u_{c} \eta_{c}}{\sigma}=\frac{Q_{c} \eta_{c}}{2 \sigma w_{c} h} .
$$

Although the laminar flow is expected due to the small length scales involved, the Reynolds number $(R e)$ is still the most frequently used dimensionless number to effectively describe microfluidics. It is a measure of the ratio of the inertial force and the viscous force

$$
R e=\frac{\rho u_{c} w_{c}}{\eta_{c}}=\frac{\rho Q_{c}}{2 \eta_{c} h} .
$$

During the process of droplet formation, the continuous phase and the dispersed phase are continuously injected with different volumetric flow rates. The ratio of flow rates $\left(Q=Q_{d} / Q_{c}\right)$ and the viscosity ratio $\left(\lambda=\eta_{d} / \eta_{c}\right)$ are two important dimensionless numbers to characterize the droplet breakup, which has been confirmed by a wide range of experimental and numerical investigations. ${ }^{18,19,23,25,26,37,57}$ The geometrical parameters, $w_{c}, w_{d}$, and $h$, lead to two additional dimensionless parameters characterizing the geometry. One is the ratio of the channel depth to the inlet width of the continuous phase $\left(\Gamma=h / w_{c}\right)$, and the other is the ratio of the inlet widths of the two phases $\left(\Lambda=w_{d} / w_{c}\right)$.

Different types of droplets, namely plug droplets, isolate droplets and satellite droplets, can be generated in the crossjunction microfluidic devices, which strongly depend on the various dimensionless parameters. ${ }^{19}$ In the present study, we focus on the formation of plug droplets for a fixed fluid pair, i.e., the viscosities of both fluids and the interfacial tension are fixed, and examine the roles of $C a, Q, \Gamma$, and $\Lambda$ in droplet formation.

\section{A. The influence of $Q$ and $\mathrm{Ca}$}

Before we study the influence of geometrical parameters, we focus on a reference cross-junction with $w_{c}=10 \delta_{x}$, $w_{d}=20 \delta_{x}$, and $h=10 \delta_{x}$, so that $\Gamma=1$ and $\Lambda=2$. We choose a fixed fluid pair with the interfacial tension $\sigma=0.06$ and the viscosity ratio $\lambda=1 / 6$. The simulations are performed in a $229 \times 61 \times 11$ lattice system and each lattice spacing $\delta_{x}$ corresponds to $10 \mu \mathrm{m}$. We examine the grid independence with several different flow conditions and find that the mesh refinement will lead to results variations not more than 5\%. Four different capillary numbers, i.e., $C a=0.002,0.004$, 0.006 , and 0.008 are used in the simulations, and the corresponding Reynolds numbers are 0.1, 0.2, 0.3, and 0.4. For each capillary number, the flow rate ratio is varied over a broad range so that the different flow patterns are observed.

As shown in Fig. 5, three typical flow patterns are identified for different flow rate ratio at a fixed capillary number. At the low flow rate ratio $Q$, the droplets are formed at the cross-junction (DCJ) due to the squeezing mechanism. When we increase $Q$, droplets are found to pinch-off at $\mathrm{DC}$, forming a thread that becomes unstable after a distance of laminar flow. This distance will increase with $Q$. As the flow rate ratio $Q$ increases to a critical value, the stable PF are observed, where the three incoming streams co-flow in parallel to the downstream without pinching. In addition, the transitions from DCJ to DC and from DC to PF are influenced by the capillary number. As $\mathrm{Ca}$ increases, the threshold value of flow rate ratio at which the transition occurs decreases, and the width of the DC regime also decreases. The different flow patterns were first observed experimentally by Guillot and Colin in a microfluidic T-junction, ${ }^{22}$ in which the transition from droplets forming at a $\mathrm{T}$-junction to stable parallel flow depends on the flow rate of both phases. Recently, Tan et $a l .{ }^{18}$ also reported the similar observations in a 


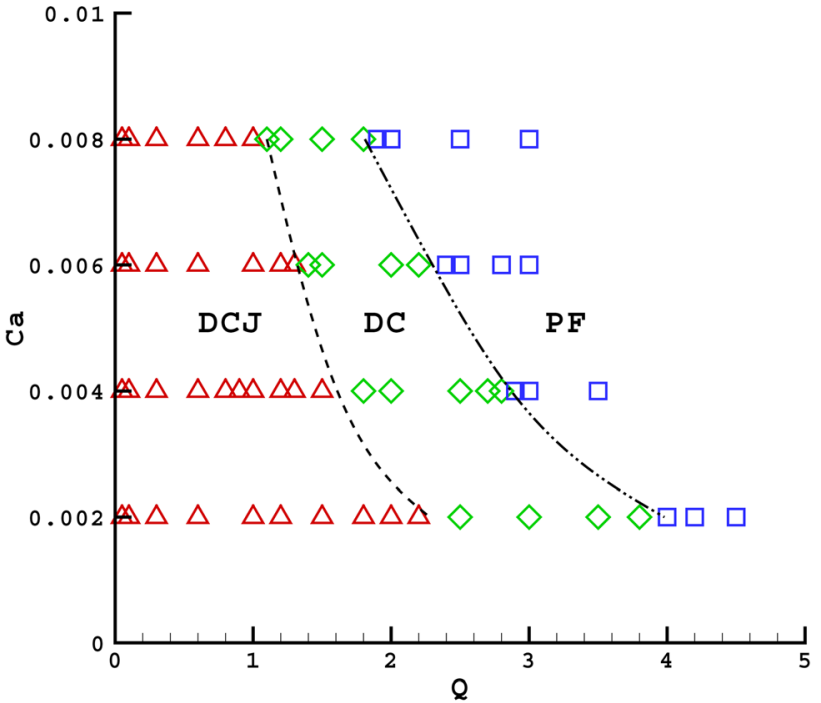

(a)
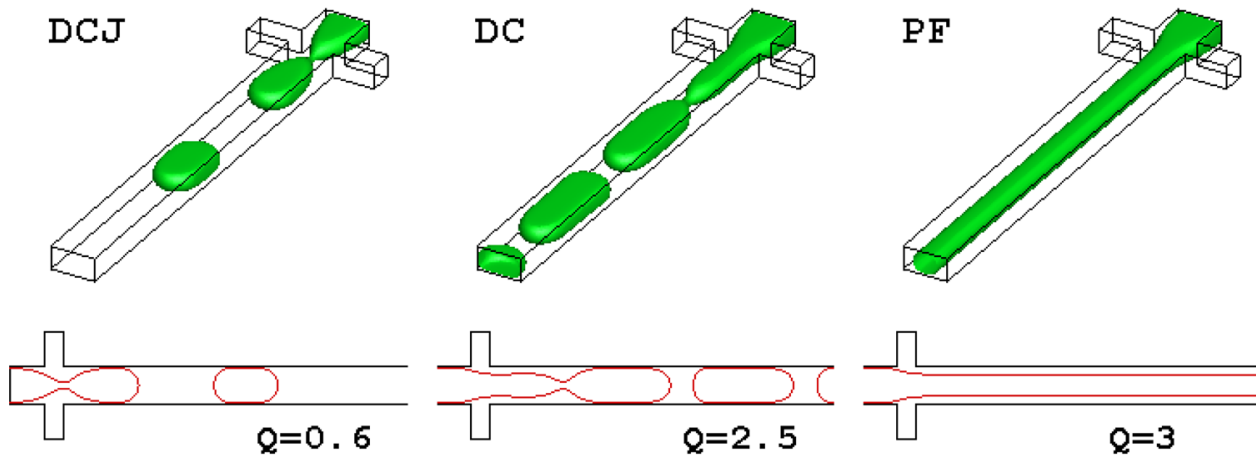

(b)

microfluidic cross-junction. In the following simulations, we will focus on the DCJ regime so that our simulations can be compared with the existing experimental work.

Based on the experimental observation of plug formation at microfluidic T-junctions, Garstecki et al. ${ }^{23}$ argued that at low $C a$, the final length of a plug is determined by two steps. First, the thread of the dispersed phase grows until it blocks the continuous phase liquid. At this critical moment, the "blocking length" of the plug is equal to the channel width, i.e., $L_{\text {block }}=w_{c}$. Afterwards, the increased pressure in the continuous phase liquid begins to "squeeze" the neck of dispersed thread. Assuming that the neck with a characteristic width $d$ squeezes at a rate approximately equal to the average velocity $\left(u_{c}\right)$ of the continuous phase. During this time, the plug continues to elongate at rate $u_{d}=\frac{Q_{d}}{w_{c} h}$ with an equivalent growth of plug volume at rate $Q_{d}$. So the "squeezing length" of the plug is $L_{\text {squeeze }} \approx \frac{d}{u_{c}} u_{d}=d Q$. Therefore, the final length $L$ of the plug can be expressed as

$$
\frac{L}{w_{c}}=\frac{L_{\text {block }}+L_{\text {squeeze }}}{w_{c}}=\frac{w_{c}+d Q}{w_{c}}=1+\omega Q,
$$

where $\omega=d / w_{c}$ is a fitting constant related to the thinning width. Their experimental data agree well with this scaling law when the constant $\omega$ is unity. Recently, Xu et al. ${ }^{29}$ compared the other experimental data and found that the
FIG. 5. (Color online) (a) Droplet flow regimes as a function of flow rate ratio $Q$ and capillary number $\mathrm{Ca}(\lambda=1 / 6$, $\Gamma=1$, and $\Lambda=2$ ), where symbols indicate different regimes $(\triangle: \mathrm{DCJ}, \diamond: \mathrm{DC}$, and $\square$ : PF) and (b) representative droplet formation at $C a=0.004$ and $Q=0.6$ (DCJ), 2.5 (DC), and $3(\mathrm{PF})$. Note that the dash and dash-dot-dot lines in (a) are to clearly distinguish the different flow regimes. "blocking length" $L_{\text {block }}$ is not always equal to $w_{c}$ but is also dependent on the channel geometry, i.e., $L_{b l o c k}=\varepsilon w_{c}$. Considering this, the scaling law, Eq. (28), can be modified as

$$
\frac{L}{w_{c}}=\varepsilon+\omega Q
$$

where $\varepsilon$ is also a fitting constant that is mainly dependent on the channel geometry.

Fig. 6 shows the formation of plugs in the DCJ regime as a function of flow rate ratio for $C a=0.002$. The
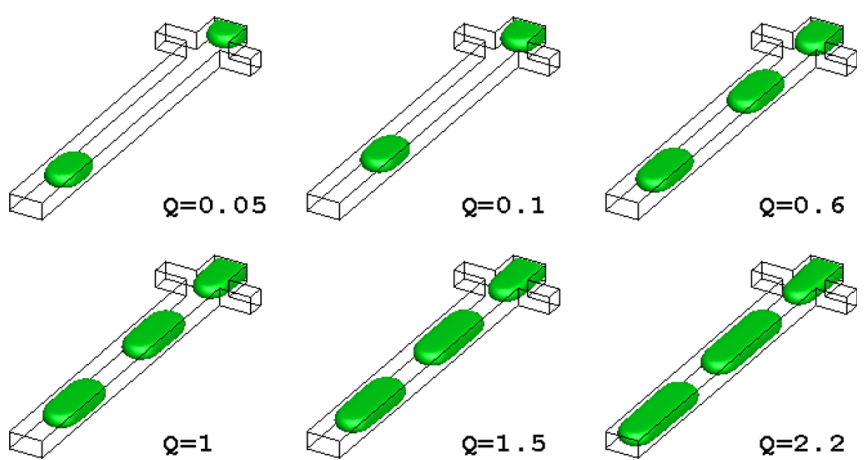

FIG. 6. (Color online) Formation of plugs as a function of flow rate ratio for $C a=0.002, R e=0.1, \lambda=1 / 6, \Gamma=1$, and $\Lambda=2$. 
monodisperse plugs are found to be regularly generated. The plug length increases with $Q$, and the formation of plugs will switch to the DC regime when $Q$ is beyond 2.2. Also, the distance between two neighboring plugs decreases when the flow rate ratio increases. Fig. 7 plots the non-dimensional length of plugs formed in the DCJ regime against the flow rate ratio for $C a=0.002,0.004,0.006$, and 0.008 . For each capillary number, our simulation results are found to obey the scaling law given by Eq. (29). Meanwhile, Eqs. (28) and (29) show that the plug lengths formed in the squeezing regime depend only on the flow rate ratio and are independent of the capillary number. However, it can be clearly seen from Fig. 7 that the fitting constants, $\varepsilon$ and $\omega$, are not solely determined by the channel geometry, and the plug lengths are indeed a function of capillary number.

In order to explore the dependence of plug length on the capillary number, we measure the plug length as a function of capillary number at a fixed flow rate ratio. Three different flow rate ratios $Q=0.1,0.3$, and 0.6 are examined, respectively. For each fixed $Q$, the capillary number is varied from 0.001 to 0.008 . It should be noted that, here, a smaller capillary number (i.e., $C a=0.001$ ) is also simulated. However, the present computational domain is not large enough to simulate the transition from $\mathrm{DC}$ to $\mathrm{PF}$ as the plug front may have moved out of the computational domain before the plug detaches from the long downstream jet in the DC regime.

Fig. 8 shows the formation of plugs as a function of capillary number for $Q=0.1$ and $Q=0.6$. For each fixed $Q$, the distance between two neighboring plugs and their size decrease with the increase of capillary number. Large flow ratio can lead to narrow distance between two neighboring plugs for the same $\mathrm{Ca}$, which is consistent with the simulation results of $C a=0.002$ as shown in Fig. 6. Fig. 9 shows the simulation results of non-dimensional length of plugs versus capillary number for three different flow rate ratios.

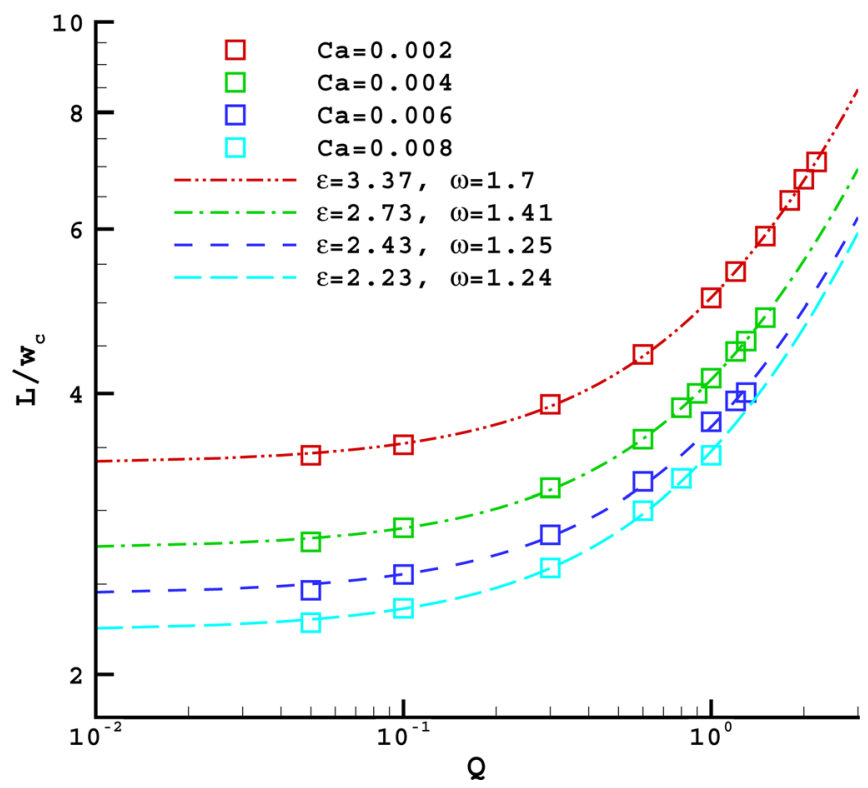

FIG. 7. (Color online) Non-dimensional length of plugs versus the flow rate ratio for $C a=\{0.002,0.004,0.006,0.008\}, \lambda=1 / 6, \Gamma=1$, and $\Lambda=2$ in the DCJ regime. Discrete symbols represent the simulation results. The lines are the fitting results of the scaling law $L / w_{c}=\varepsilon+\omega Q$.
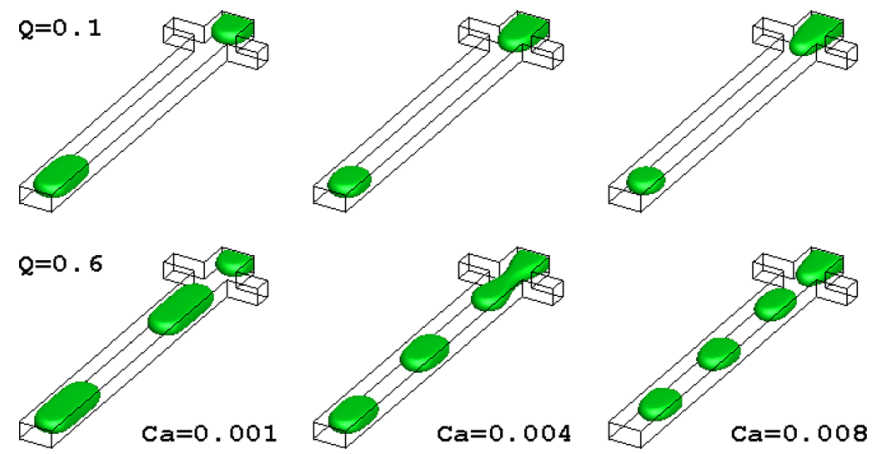

FIG. 8. (Color online) Formation of plugs as a function of the capillary number for $Q=\{0.1,0.6\}, \lambda=1 / 6, \Gamma=1$, and $\Lambda=2$ in the DCJ regime.

Clearly, the non-dimensional length of plugs $\left(L / w_{c}\right)$ shows no sign of approaching a constant value as the capillary number decreases and exhibits a power-law dependence on the capillary number, i.e., $L / w_{c}=k C a^{m}$, which is independent of the flow rate ratio $Q$. The power-law behavior was also experimentally observed by Tan and his co-workers in microfluidic cross-junction ${ }^{18}$ and T-junction, ${ }^{58}$ where the formation of plugs occurred in the squeezing (DCJ) regime. Christopher et al. $^{26}$ found that, in both squeezing and dripping regimes, the droplet volume exhibits a power-law dependence on the capillary number with a power-law exponent approximately equal to 0.3 at low viscosity ratio $(\lambda \leq 1 / 50)$.

Considering the influence of capillary number and flow rate ratio, the generated plug length can be predicted by

$$
\frac{L}{w_{c}}=(\tilde{\varepsilon}+\tilde{\omega} Q) C a^{\tilde{m}},
$$

where $\tilde{\varepsilon}, \tilde{\omega}$, and $\tilde{m}$ are the fitting parameters that mainly depend on the channel geometry. All of our numerical

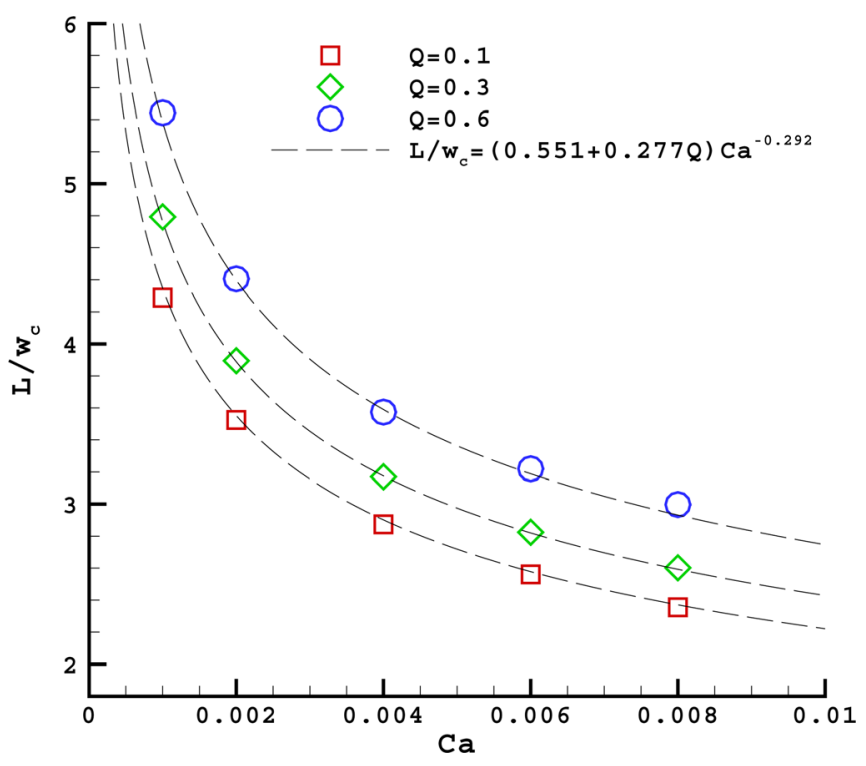

FIG. 9. (Color online) Non-dimensional length of plugs versus the capillary number for $Q=\{0.1,0.3,0.6\}, \lambda=1 / 6, \Gamma=1$, and $\Lambda=2$ in the DCJ regime. Discrete symbols represent the simulation results. Dashed-lines represent the scaling law $\frac{L}{w_{c}}=(\tilde{\varepsilon}+\tilde{\omega} Q) C a^{\tilde{m}}$ by fitting the simulation results. 
simulations confirm this scaling law and we can determine the coefficients as

$$
\frac{L}{w_{c}}=(0.551+0.277 Q) C a^{-0.292} .
$$

To test our scaling law, the parity plot is used in which the predicted plug length is calculated by Eq. (30) and plotted as a function of our simulation results. The match between model predictions and simulation results is indicated by the scatter of data around the line of parity. The closer the data points are to the line of parity and the more even their distribution is, the better model predictions and simulations match. The predicted results of Eq. (30) show good agreement with our numerical simulations, as shown in Fig. 10. The fitting power-law exponent $\tilde{m}=-0.292$ is very close to the experimental findings of Chiristopher et al. ${ }^{26}$ in a T-junction. Through the statistical analysis on the droplet formation in microfluidic T-junctions, Steegmans et al. ${ }^{30}$ found that none of the available scaling laws is general enough to describe the original data and the data from other literature sources. A two-step model was found to be statistically valid over the whole range of literature data. In their two-step model, the final droplet volume $V$ is a result of two-stage growth, which is similar to the idea of Garstecki et al. ${ }^{23}$ Initially, the droplet grows to a critical volume $V_{c}$ until the forces exerted on the interface become balanced. Subsequently, the droplet continues to grow for a time $t_{n}$ until final pinches-off, due to the continuous injection of the dispersed phase fluid, so that the final droplet volume becomes

$$
V=V_{c}+t_{n} Q_{d}
$$

Similar to Steegmans et al., ${ }^{30}$ we also assume plugs enclosed between channel walls to be flat ellipses, so that Eq. (30) can be expressed as

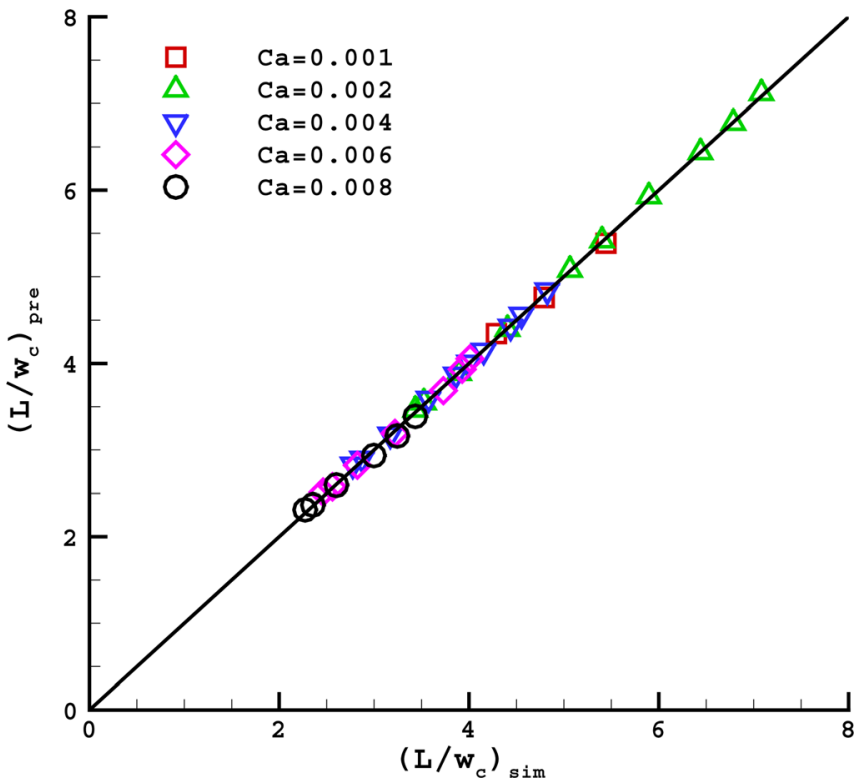

FIG. 10. (Color online) The parity plot of the non-dimensional length of plugs between the correlated results $\left(L / w_{c}\right)_{\text {pre }}$ from Eq. (31) and the simulation results $\left(L / w_{c}\right)_{\text {sim }}$ for $C a=0.001,0.002,0.004,0.006$, and 0.008 . The solid line represents the line of parity.

$$
V=\frac{\pi w_{c} w_{d} h}{4}\left(\tilde{\varepsilon}+\frac{\tilde{\omega}}{Q_{c}} Q_{d}\right) C a^{\tilde{m}} .
$$

It can be clearly seen that our scaling law given by Eq. (30) is consistent with the two-step model, i.e., Eq. (32) if

$$
V_{c}=\frac{\pi}{4} w_{c} w_{d} h \tilde{\varepsilon} C a^{\tilde{m}}, \quad t_{n}=\frac{\pi}{4 Q_{c}} w_{c} w_{d} h \tilde{\omega} C a^{\tilde{m}}
$$

It is obvious that the necking time $t_{n}$ is associated with both $C a$ and $Q_{c}$. At a fixed capillary number, a large shear rate of continuous phase is expected to shorten the necking time, leading to smaller droplets.

\section{B. The influence of $\Lambda$ and $\Gamma$}

To test whether the confinement of geometry plays an important role in the breakup of plugs, ${ }^{23,26,29}$ we examine the influence of the dimensionless geometrical parameters $\Lambda$ and $\Gamma$ on the length and shapes of the plugs. In the simulations, to single out the influence of $\Lambda, w_{c}$ and $h$ are kept constant with $\Gamma=5 / 8$. To study the influence of $\Gamma, w_{c}$ and $w_{d}$ are kept constant with $\Lambda=1$. The fluid pair is fixed with the interfacial tension $\sigma=0.06$ and the viscosity ratio $\lambda=0.3$. To statistically obtain the scaling law given by Eq. (30) for each group of geometrical parameters, we carry out the numerical simulations with $C a=0.001,0.0018$, and 0.004 , and the corresponding Reynolds number is $0.211,0.38$, and 0.844 , respectively. For each fixed $\mathrm{Ca}$, we vary the flow rate ratio, i.e., $Q=\{0.05,0.1,0.25,0.5,1,1.5\}$ by varying the flow rate of the dispersed phase.

Fig. 11 shows the formation of plugs in the DCJ regime as a function of width ratio $(\Lambda)$ at $C a=0.0018, R e=0.38$, and $Q=\{0.05,0.25,0.5,1.5\}$. Three different width ratios are examined, i.e., $\Lambda=1,1.5$, and 2 , with the computational domain consisting of $250 \times 57 \times 11,250 \times 65 \times 11$, and $250 \times 73 \times 11$ lattices, respectively. For each fixed flow rate ratio, the plug length increases with $\Lambda\left(w_{d}\right)$, which corresponds to a more significant increase in the volume of plugs. Also, the increasing $\Lambda$ can enlarge the distance between two neighboring plugs. For each fixed width ratio, big flow rate ratio can lead to large plugs and narrow distance between consecutive plugs. We also notice that the plugs become
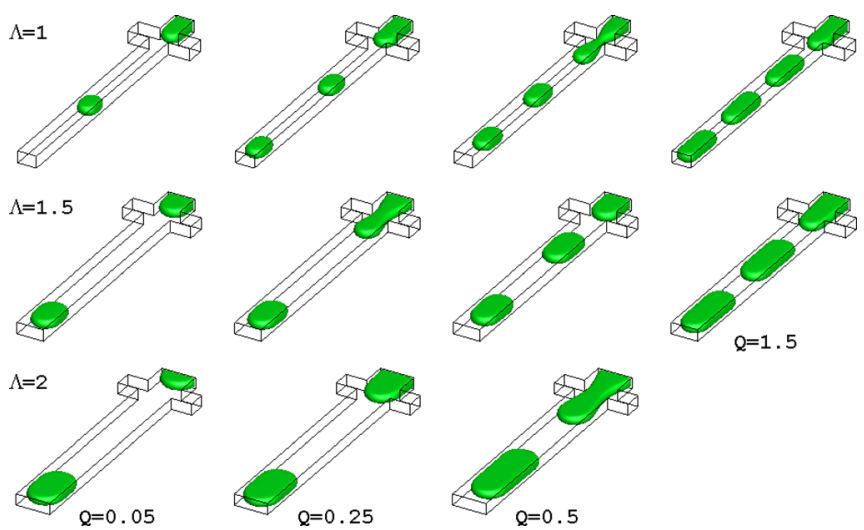

$Q=1.5$

FIG. 11. (Color online) Formation of plugs as a function of the width ratio at various flow rate ratios in the DCJ regime with $C a=0.0018, R e=0.38$, $\lambda=0.3$, and $\Gamma=5 / 8$. 
more and more difficult to "pinch-off" as the width ratio increases. For large values of $\Lambda$ and $Q$, e.g., $\Lambda=2$ and $Q=1.5$, we find that the detachment point moves towards the downstream of the junction, so the DC regime starts. Therefore, it can be expected that transitions from DCJ to DC and from DC to PF occur at smaller flow rate ratios for a large $\Lambda$.

The scaling law given in Eq. (30) is used to fit our numerical simulation results for $\Lambda=1,1.5$, and 2. Based on the least-square fitting, the resulting scaling equations can be expressed as $\frac{L}{w}=(0.32+0.219 Q) C a^{-0.243}$ for $\Lambda=1$, $\frac{L}{w_{c}}=(0.397+0.21 Q) C a^{-0.28}$ for $\Lambda=1.5$, and $\frac{L}{w_{c}}=(0.788$ $+0.521 Q) \mathrm{Ca}^{-0.222}$ for $\Lambda=2$. Fig. 12 gives the comparison of non-dimensional length of plugs between our simulation results and the predicted results for $\Lambda=1,1.5$, and 2 . It can be clearly seen that the simulation results could be well described by our scaling law with the fitting parameters depending on the width ratio. Fig. 13 plots the non-dimensional length of plugs as a function of flow rate ratio at $C a=0.0018, R e=0.38$, and $\Gamma=5 / 8$ for three different width ratios. With the fixed $C a$ of 0.0018 , our scaling law, i.e., Eq. (30) reduces to Eq. (29). Specifically, they become $\frac{L}{w_{c}}=1.483+1.015 Q$ for $\Lambda=1, \frac{L}{w_{c}}=2.328+1.232 Q$ for $\Lambda=1.5$, and $\frac{L}{w_{c}}=3.202+2.115 Q$ for $\Lambda=2$, respectively. We can easily observe that the fitting constants $\varepsilon$ and $\omega$ in Eq. (29) are both dependent on the width ratio $\Lambda$. Also, the values of $\varepsilon$ and $\omega$ increase as $\Lambda$ increases.

Fig. 14 shows the non-dimensional length of plugs as a function of the width ratio for $C a=0.0018$ and 0.0036. Consistent with the previous findings (see Figs. 7-9), the plug length decreases with the increase of $C a$ for the fixed $\Lambda$ and $Q$. At small width ratio $(\Lambda<1)$, the plug length is weakly dependent on the width ratio. When the width ratio is beyond 1 , i.e., $\Lambda>1$, the plug length is approximately linearly proportional to the width ratio. In addition, the simulation

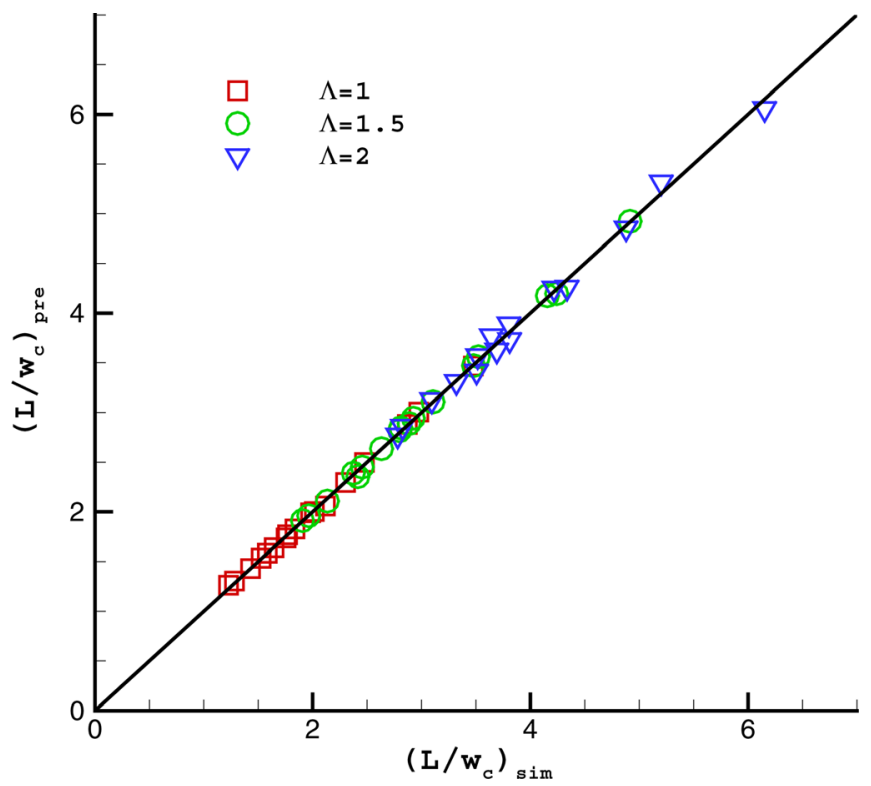

FIG. 12. (Color online) The parity plot of non-dimensional length of plugs between the predicted results $\left(L / w_{c}\right)_{\text {pre }}$ from Eq. (30) and the simulation results $\left(L / w_{c}\right)_{\operatorname{sim}}$ for $\Lambda=1,1.5$, and 2 . The solid line represents the line of parity. The values of the fitting parameters in Eq. (30) are listed in the text.

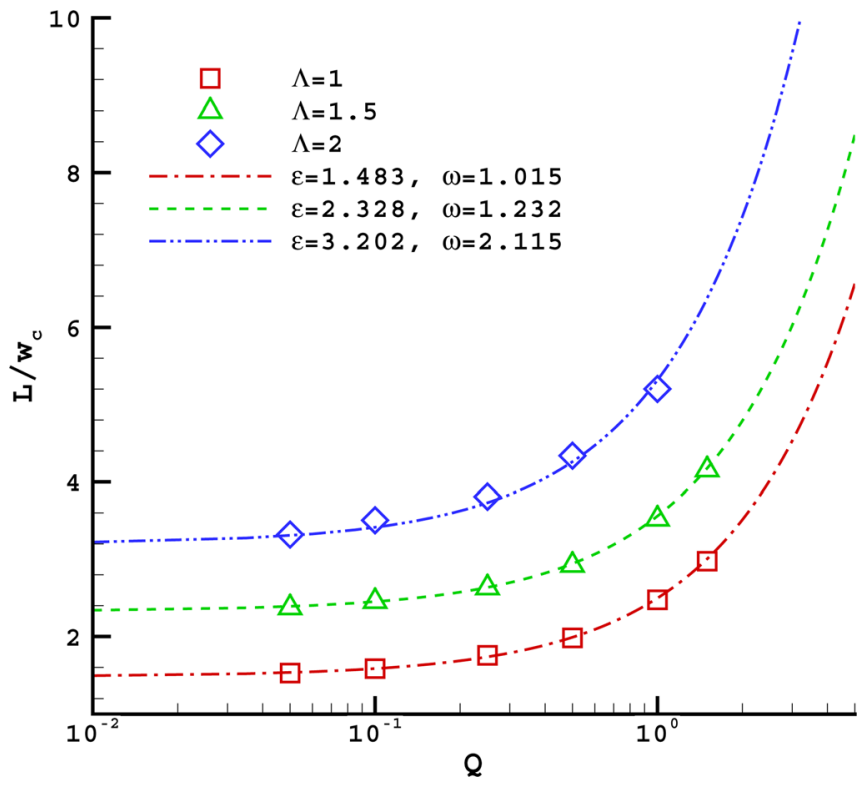

FIG. 13. (Color online) The non-dimensional length of plugs versus the flow rate ratio for $\Lambda=\{1,1.5,2\}, C a=0.0018, R e=0.38$, and $\Gamma=5 / 8$ in the DCJ regime. The discrete symbols are the simulation results. The lines are the fitting results of the scaling law $L / w_{c}=\varepsilon+\omega Q$.

results shown in Fig. 14 are in good agreement with the experimental findings of Christopher et $_{\text {al. }}{ }^{26}$ for droplet generation at T-junctions. Fig. 15 gives the comparison of plug shapes for a broad range of width ratios with $Q=0.2$ and $C a=0.0036$. It can be easily found that the width ratio can significantly influence the volume of plugs. The volume of plugs increases with the width ratio. Also, the increasing width ratio can enlarge the distance between two neighboring plugs for $\Lambda>1$.

We also investigate the influence of $\Gamma$ on the formation of plugs at a fixed width ratio $\Lambda=1$. We choose

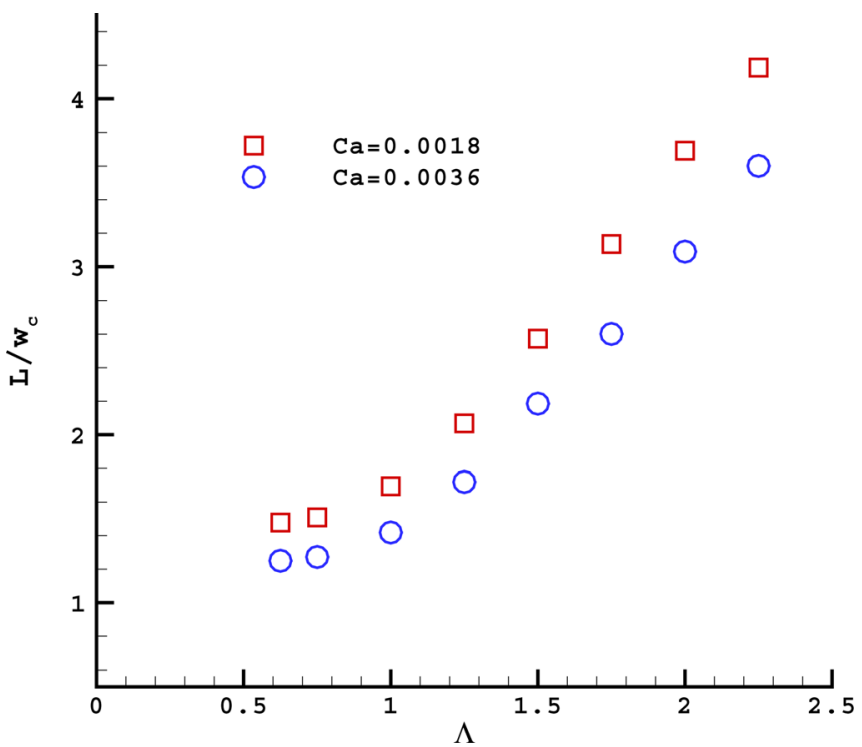

FIG. 14. (Color online) The non-dimensional length of plugs versus the width ratio at a fixed flow rate ratio $Q=0.2$ and a fixed viscosity ratio $\lambda=0.3$ for two different capillary numbers. The width ratio is adjusted by changing the magnitude of the dispersed-phase channel width while the continuous-phase channel width is kept fixed. 

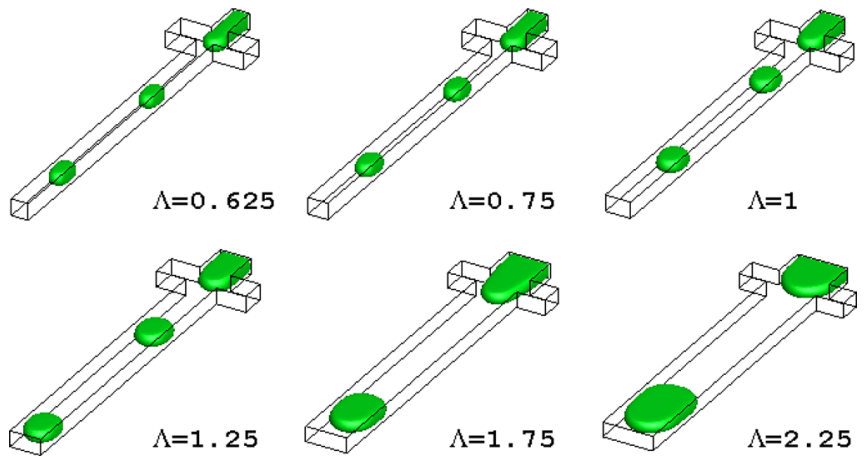

FIG. 15. (Color online) Comparison of plug shapes for a broad range of width ratio with $Q=0.2, C a=0.0036, R e=0.76, \lambda=0.3$, and $\Gamma=5 / 8$ in the DCJ regime.

$\Gamma=\{5 / 8,1,5 / 4\}$ with the computational domain consisting of $250 \times 57 \times 11,250 \times 57 \times 17$, and $250 \times 57 \times 21$ lattices, respectively. Fig. 16 shows the simulation results for $\Gamma=\{5 / 8,1,5 / 4\}$ at $C a=0.0018$ and $R e=0.38$ with a range of flow rate ratios. For each fixed flow rate ratio, the size of plugs increases as $\Gamma$ increases. In the same way, we use our scaling law (i.e., Eq. (30)) to fit the simulation data for each fixed $\Gamma$. The calculated scaling equations could be written as $\frac{L}{w}=(0.32+0.219 Q) \mathrm{Ca}^{-0.243}$ for $\Gamma=5 / 8, \quad \frac{L}{w_{c}}=(0.347+0.253 Q) C a^{-0.245}$ for $\Gamma=1$, and $\frac{L}{w_{c}}=(0.36+0.27 Q) C a^{-0.255}$ for $\Gamma=5 / 4$. The predicted results of these scaling equations all agree well with the simulation results, as can be shown in Fig. 17. This indicates again that our scaling law is general enough to describe the size of plugs formed in the DCJ regime at microfluidic cross-junctions.

Fig. 18 shows the non-dimensional droplet length as a function of the flow rate ratio at $C a=0.0018, R e=0.38$, and $\Lambda=1$ for three different $\Gamma$. For each fixed channel depth, the non-dimensional length of plugs increases with the flow rate ratio, as observed in the previous cases. For the given capillary number, i.e., $C a=0.0018$, the calculated scaling equations can reduce to $\frac{L}{w_{c}}=1.483+1.015 Q$ for $\Gamma=5 / 8$, $\frac{L}{w_{c}}=1.634+1.191 Q$ for $\Gamma=1$, and $\frac{L}{w_{c}}=1.807+1.353 Q$ for $\Gamma=5 / 4$. Obviously, both fitting parameters $\varepsilon$ and $\omega$ depend on $\Gamma$, and they both increase as $\Gamma$ increases. A similar observation was reported by Gupta and Kumar ${ }^{59}$ in microflui-
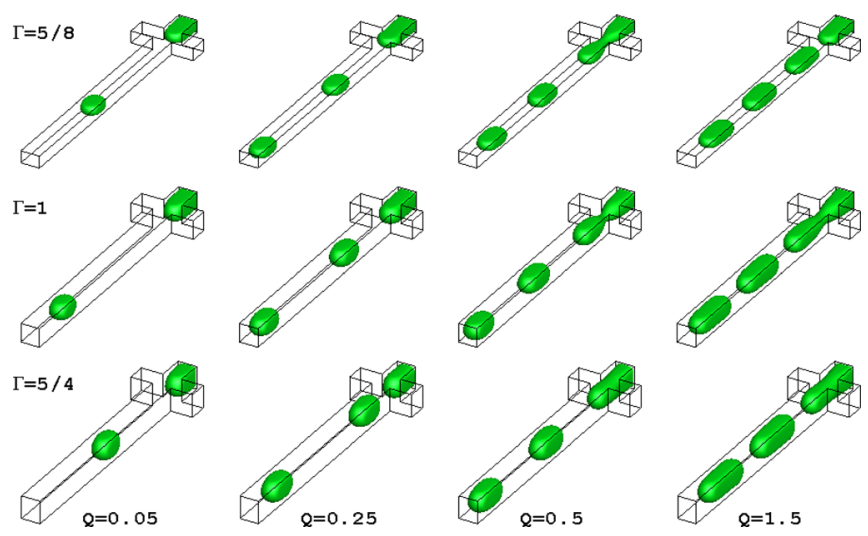

FIG. 16. (Color online) Formation of plugs as a function of height-to-width ratio for $Q=\{0.05,0.25,0.5,1.5\}$ with $C a=0.0018, R e=0.38, \lambda=0.3$, and $\Lambda=1$ in the DCJ regime.

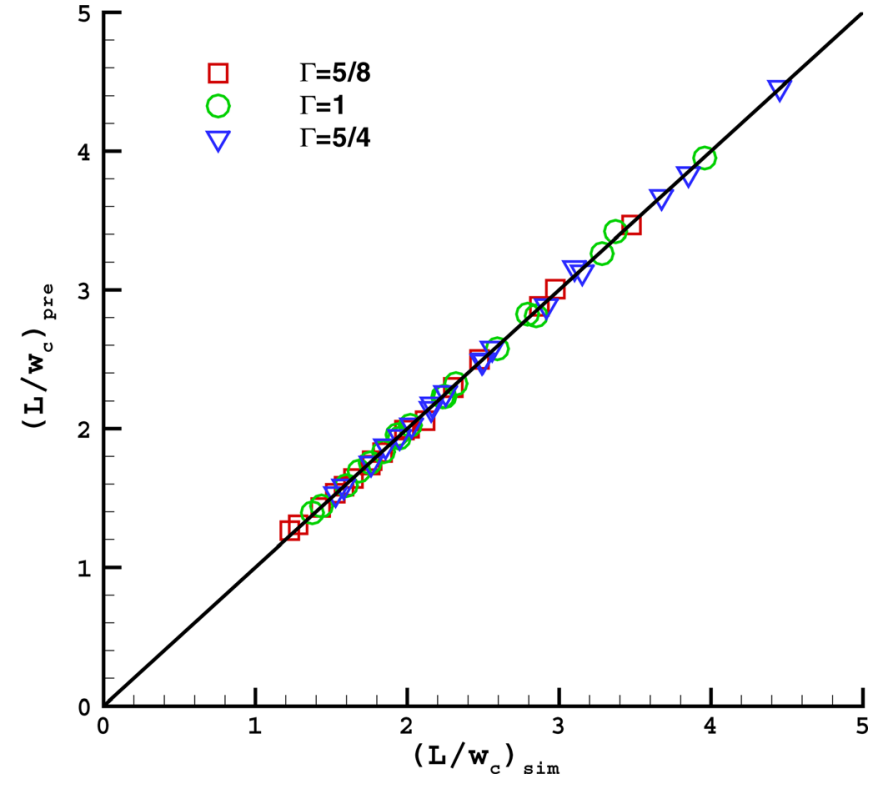

FIG. 17. (Color online) Parity plot of the non-dimensional length of plugs between the predicted results $\left(L / w_{c}\right)_{\text {pre }}$ from Eq. (30) and the simulation results $\left(L / w_{c}\right)_{\text {sim }}$ for $\Gamma=5 / 8,1$, and $5 / 4$. The solid line represents the line of parity. The values of the fitting parameters in Eq. (30) are listed in the text.

dic T-junctions. Our simulation results shown in Fig. 18 also indicate that the droplet behavior is expected to approach the scaling law of Eq. (28) as the channel depth $h$ decreases.

Although the scaling law of Eq. (30) developed from our simulation results is consistent with some experimental findings, we would like to more directly compare our simulations results with experimental data. Wu et al. ${ }^{36}$ experimentally reported droplet generation in a microfluidic cross-junction, where, unlike the other experimental work, they clearly gave

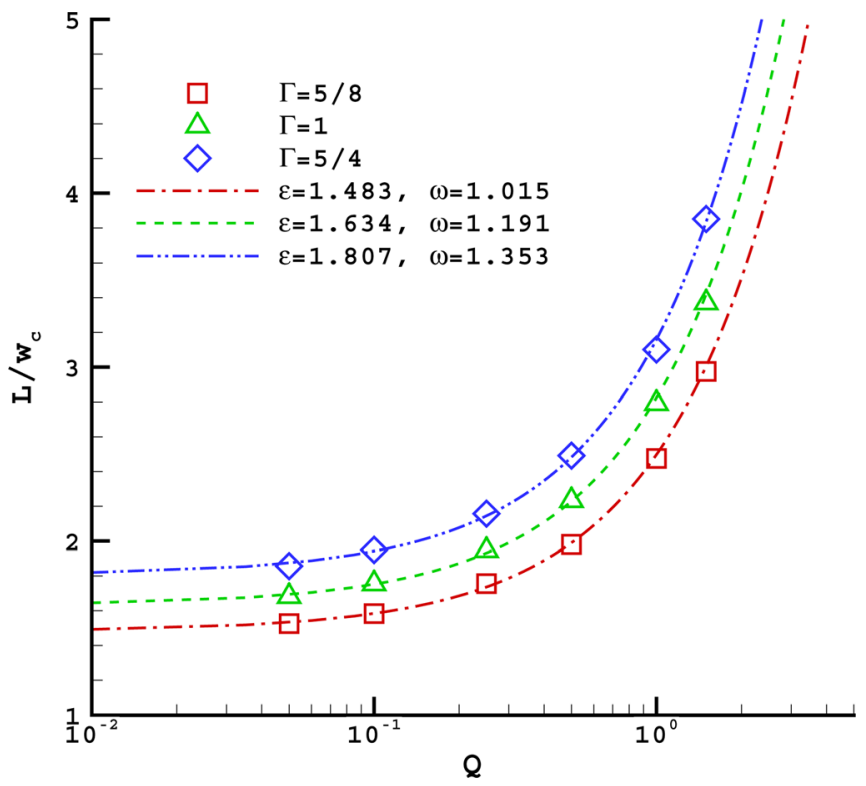

FIG. 18. (Color online) The non-dimensional length of plugs versus the flow rate ratio at $C a=0.0018, R e=0.38, \lambda=0.3$, and $\Lambda=1$ for three different height-to-width ratios. The height-to-width ratio $\Gamma$ is adjusted by changing the magnitude of the channel depth, while the channel widths are kept the same. The discrete symbols are the simulation results. The lines are the fitting results of the scaling law $L / w_{c}=\varepsilon+\omega Q$. 
TABLE I. Non-dimensional droplet length obtained from the experiment (Ref. 36) and our simulations with $\rho_{S}^{N}=-1$ and -0.75 at various flow rate ratios and capillary numbers. For comparison, we also give the predicted values of non-dimensional droplet length using the scaling equation $\frac{L}{w_{c}}=(0.645+0.332 Q) C a^{-0.218}$. Note: our definition of $C a$ is different from Ref. 36.

\begin{tabular}{llllll}
\hline \hline & & \multicolumn{5}{c}{ Droplet length $\left(L / w_{c}\right)$} \\
\cline { 3 - 6 }$Q$ & \multicolumn{1}{c}{$\mathrm{Ca}$} & $\mathrm{LB}\left(\rho_{S}^{N}=-1\right)$ & $\mathrm{LB}\left(\rho_{S}^{N}=-0.75\right)$ & Exp. & Pre. \\
\hline $1 / 6$ & 0.002 & 3.12 & 2.73 & 2.75 & 2.7 \\
$1 / 3$ & 0.002 & 3.42 & 2.92 & 3 & 2.92 \\
$1 / 2$ & 0.002 & 3.69 & 3.08 & 3.1 & 3.13 \\
1 & 0.002 & 4.58 & 3.79 & 4.1 & 3.77 \\
$1 / 6$ & 0.0028 & 2.87 & 2.52 & 2.56 & 2.51 \\
$1 / 6$ & 0.004 & 2.63 & 2.33 & 2.25 & 2.33 \\
$1 / 6$ & 0.0081 & 2.18 & 1.99 & 2 & 2 \\
\hline \hline
\end{tabular}

the most of required physical and mechanical parameters for meaningful comparison. Our numerical simulations are performed with $\rho_{S}^{N}=-1$ and -0.75 specified at the solid walls, corresponding to the static contact angle $\theta \approx 180^{\circ}$ and $160^{\circ}$, respectively. The other parameters are set to be the same as in Ref. 36. Table I shows that the simulation results with $\rho_{S}^{N}=-0.75$ are in good agreement with the experimental findings, while the simulations with $\rho_{S}^{N}=-1$ produce larger droplets. We also find that the droplet sizes obtained by our simulations can be described by Eq. (30) as $\frac{L}{w_{c}}=(0.58$ $+0.358 Q) C a^{-0.255}$ for $\rho_{S}^{N}=-1$ and $\frac{L}{w_{c}}=(0.645+0.332 Q)$ $C a^{-0.218}$ for $\rho_{S}^{N}=-0.75$. For comparison, Table I also gives the predicted results of non-dimensional droplet length using the scaling equation $\frac{L}{w_{c}}=(0.645+0.332 Q) \mathrm{Ca}^{-0.218}$. Obviously, the predicted results coincide with our simulation results $\left(\rho_{S}^{N}=-0.75\right)$ and the experimental data of $\mathrm{Wu}$ et al. ${ }^{36}$ This indicates that our proposed scaling law can predict the correct droplet size generated in microfluidic cross-junctions.

Finally, we note that the droplet size exhibits a powerlaw dependence on the capillary number with the fitted power-law exponent ranging from -0.3 to -0.2 for all the flow conditions and channel geometries in the present study. As we stated above, this power-law behavior has also been observed by some other authors. However, the values of the power-law exponent vary significantly in their observations. For example, Tan et al. ${ }^{18}$ and $\mathrm{Xu}$ et al. ${ }^{29}$ experimentally found that the plug length obeys $\frac{L}{w_{c}} \propto \mathrm{Ca}^{-0.2}$ in a microfluidic cross-junction and a microfluidic $\mathrm{T}$-junction, while van der Graaf et $a l .{ }^{24}$ numerically observed that, in both confined and unconfined droplet breakup at a T-junction, the final droplet volume $V$ could be expressed as $V \propto \mathrm{Ca}^{-0.75}$. Christopher et $a .^{26}$ recently reported that the droplet volume $V$ approximately follows $\mathrm{V} \propto \mathrm{Ca}^{-0.3}$ in the microfluidic T-junctions with $\Gamma<1$ and the viscosity ratio $\lambda \leq 1 / 50$. Herein, it should be noted that the droplet size is often expressed as volume $V$ which can be correlated to the plug length as $V \propto \frac{L}{w_{c}}$ for the plugs enclosed in a microchannel.

\section{CONCLUSIONS}

The 3D lattice Boltzmann model has been applied to study the droplet formation in microfluidic cross-junctions for low capillary numbers $(\mathrm{Ca}<0.01)$. Three different flow regimes as a consequence of interaction between two immiscible fluids are identified to be dependent on the capillary number and the flow rates of the continuous and dispersed phases. A regime map is created to describe the transition from droplets formed at a cross-junction (DCJ), downstream of the cross-junction (DC), to stable PF. The influence of flow rate ratio, capillary number, and dimensionless geometrical parameters $(\Lambda$ and $\Gamma$ ) is studied in detail with a broad range of flow conditions in the DCJ regime. The formation of plugs in the DCJ regime is shown to be dominated by the build-up of pressure that arises in the upstream when the emerging droplet interface obstructs the main channel. We observe that the length of the plugs is linearly proportional to the flow rate ratio for a fixed capillary number and exhibits a power-law dependence on the capillary number for a fixed flow rate ratio. Considering the effects of capillary number and flow rate ratio, an empirical scaling law is developed to predict the length of plugs. It is consistent with the two-step models proposed by van der Graaf et al. ${ }^{60}$ and Steegmans et al. ${ }^{30}$ The channel geometry is found to play an important role in the process of plug breakup since the squeezing pressure becomes significant when the emerging dispersed-interface obstructs the main channel. At a fixed capillary number, our scaling law can reduce to the scaling equation proposed by $\mathrm{Xu}$ et al., ${ }^{29}$ i.e., $L / w_{c}=\varepsilon+\omega Q$. We find that the width ratio $(\Lambda)$ and the depth-to-width ratio $(\Gamma)$ can affect the fitting constants $\varepsilon$ and $\omega$. Also, we find that the threshold value of flow rate ratio decreases with the width ratio, which distinguishes the different flow regimes.

\section{ACKNOWLEDGMENTS}

The authors would like to thank Dr. Long Wu for the valuable discussions and Dr. Ian Halliday for providing us a version of his two-dimensional LB code.

${ }^{1}$ D. Beebe, M. Wheeler, H. Zeringue, E. Walters, and S. Raty, "Microfluidic technology for assisted reproduction," Theriogenology 57, 125 (2002).

${ }^{2}$ A. J. deMello, "Microfluidics: DNA amplification moves on," Nature 422, 28 (2003).

${ }^{3}$ G. F. Christopher and S. L. Anna, "Microfluidic methods for generating continuous droplet streams," J. Phys. D: Appl. Phys. 40, R319 (2007).

${ }^{4}$ A. Huebner, S. Sharma, M. Srisa-Art, F. Hollfelder, J. B. Edel, and A. J. deMello, "Microdroplets: A sea of applications?," Lab Chip 8, 1244 (2008).

${ }^{5}$ B. Zheng, L. S. Roach, and R. F. Ismagilov, "Screening of protein crystallization conditions on a microfluidic chip using nanoliter-size droplets," J. Am. Chem. Soc. 125, 11170 (2003).

${ }^{6}$ H. Song, D. L. Chen, and R. F. Ismagilov, "Reactions in droplets in microfluidic channels,” Angew. Chem. Int. Ed. 45, 7336 (2006).

${ }^{7}$ S. Sugiura, M. Nakajima, H. Itou, and M. Seki, "Synthesis of polymeric microspheres with narrow size distributions employing microchannel emulsification," Macromol. Rapid Commun. 22, 773 (2001).

${ }^{8}$ D. Dendukuri, K. Tsoi, T. A. Hatton, and P. S. Doyle, "Controlled synthesis of nonspherical microparticles using microfluidics," Langmuir 21, 2113 (2005).

${ }^{9}$ V. Srinivasan, V. K. Pamula, and R. B. Fair, "Droplet-based microfluidic lab-on-a-chip for glucose detection,” Anal. Chim. Acta 507, 145 (2004).

${ }^{10}$ J. Khandurina, T. E. McKnight, S. C. Jacobson, L. C. Waters, R. S. Foote, and J. M. Ramsey, "Integrated system for rapid PCR-based DNA analysis in microfluidic devices," Anal. Chem. 72, 2995 (2000).

${ }^{11}$ N. Bontoux, A. Pépin, Y. Chen, A. Ajdari, and H. A. Stone, "Experimental characterization of hydrodynamic dispersion in shallow microchannels," Lab Chip 6, 930 (2006). 
${ }^{12}$ J. D. Tice, H. Song, A. D. Lyon, and R. F. Ismagilov, "Formation of droplets and mixing in multiphase microfluidics at low values of the Reynolds and the Capillary numbers," Langmuir 19, 9127 (2003).

${ }^{13}$ M. Yasuno, S. Sugiura, S. Iwamoto, M. Nakajima, A. Shono, and K. Satoh, "Monodispersed microbubble formation using microchannel technique," AIChE J. 50, 3227 (2004).

${ }^{14}$ S. Sugiura, M. Nakajima, and M. Seki, "Prediction of droplet diameter for microchannel emulsification: Prediction model for complicated microchannel geometries," Ind. Eng. Chem. Res. 43, 8233 (2004).

${ }^{15}$ S. L. Anna, N. Bontoux, and H. A. Stone, "Formation of dispersions using 'flow focusing' in microchannels," Appl. Phys. Lett. 82, 364 (2003).

${ }^{16}$ T. Cubaud, M. Tatineni, X. Zhong, and C.-M. Ho, "Bubble dispenser in microfluidic devices,” Phys. Rev. E 72, 037302 (2005).

${ }^{17}$ P. Garstecki, H. A. Stone, and G. M. Whitesides, "Mechanism for flowrate controlled breakup in confined geometries: A route to monodisperse emulsions," Phys. Rev. Lett. 94, 164501 (2005).

${ }^{18}$ J. Tan, J. Xu, S. Li, and G. Luo, "Drop dispenser in a cross-junction microfluidic device: Scaling and mechanism of break-up," Chem. Eng. J. 136, 306 (2008).

${ }^{19}$ T. Fu, Y. Ma, D. Funfschilling, and H. Z. Li, "Bubble formation and breakup mechanism in a microfluidic flow-focusing device," Chem. Eng. Sci. 64(10), 2392 (2009).

${ }^{20}$ T. Thorsen, R. W. Roberts, F. H. Arnold, and S. R. Quake, "Dynamic pattern formation in a vesicle-generating microfluidic device," Phys. Rev. Lett. 86, 4163 (2001).

${ }^{21}$ T. Nisisako, T. Torii, and T. Higuchi, "Droplet formation in a microchannel network," Lab Chip 2, 24 (2002).

${ }^{22} \mathrm{P}$. Guillot and A. Colin, "Stability of parallel flows in a microchannel after a T junction," Phys. Rev. E 72, 066301 (2005).

${ }^{23}$ P. Garstecki, M. J. Fuerstman, H. A. Stone, and G. M. Whitesides, "Formation of droplets and bubbles in a microfluidic T-junction-scaling and mechanism of break-up," Lab Chip 6, 437 (2006).

${ }^{24}$ S. van der Graaf, T. Nisisako, C. G. P. H. Schroën, R. G. M. van der Sman, and R. M. Boom, "Lattice Boltzmann simulations of droplet formation in a T-shaped microchannel," Langmuir 22, 4144 (2006).

${ }^{25}$ M. D. Menech, P. Garstecki, F. Jousse, and H. A. Stone, "Transition from squeezing to dripping in a microfluidic T-shaped junction," J. Fluid Mech. 595, 141 (2008).

${ }^{26}$ G. F. Christopher, N. N. Noharuddin, J. A. Taylor, and S. L. Anna, "Experimental observations of the squeezing-to-dripping transition in T-shaped microfluidic junctions," Phys. Rev. E 78, 036317 (2008).

${ }^{27}$ P. B. Umbanhowar, V. Prasad, and D. A. Weitz, "Monodisperse emulsion generation via drop break off in a coflowing stream," Langmuir 16, 347 (2000).

${ }^{28}$ J. Hua, B. Zhang, and J. Lou, "Numerical simulation of microdroplet formation in coflowing immiscible liquids," AIChE J. 53, 2534 (2007).

${ }^{29}$ J. Xu, S. Li, J. Tan, and G. Luo, "Correlations of droplet formation in T-junction microfluidic devices: From squeezing to dripping," Microfluid. Nanofluid. 5, 711 (2008).

${ }^{30}$ M. Steegmans, C. Schron, and R. Boom, "Generalised insights in droplet formation at T-junctions through statistical analysis," Chem. Eng. Sci. 64(13), 3042 (2009).

${ }^{31}$ W. Shyy, R. W. Smith, H. S. Udaykumar, and M. M. Rao, Computational Fluid Dynamics with Moving Boundaries (Taylor \& Francis, Washington, DC, 1996).

${ }^{32}$ S. Succi, The Lattice Boltzmann Equation for Fluid Dynamics and Beyond (Oxford University Press, Oxford, 2001).

${ }^{33} \mathrm{X}$. He and L.-S. Luo, "A priori derivation of the lattice Boltzmann equation,” Phys. Rev. E 55, R6333 (1997).

${ }^{34}$ M. M. Dupin, I. Halliday, and C. M. Care, "Simulation of a microfluidic flow-focusing device," Phys. Rev. E 73, 055701 (2006).

${ }^{35}$ Z. Yu, O. Hemminger, and L.-S. Fan, "Experiment and lattice Boltzmann simulation of two-phase gas-liquid flows in microchannels," Chem. Eng. Sci. 62, 7172 (2007).
${ }^{36}$ L. Wu, M. Tsutahara, L. S. Kim, and M. Ha, "Three-dimensional lattice Boltzmann simulations of droplet formation in a cross-junction microchannel," Int. J. Multiphase Flow 34, 852 (2008).

${ }^{37}$ H. Liu and Y. Zhang, "Droplet formation in a T-shaped microfluidic junction,” J. Appl. Phys. 106, 034906 (2009).

${ }^{38}$ S. Chen and G. D. Doolen, "Lattice Boltzmann method for fluid flows," Annu. Rev. Fluid Mech. 30(1), 329 (1998).

${ }^{39}$ A. K. Gunstensen, D. H. Rothman, S. Zaleski, and G. Zanetti, "Lattice Boltzmann model of immiscible fluids," Phys. Rev. A 43(8), 4320 (1991).

${ }^{40} \mathrm{X}$. Shan and H. Chen, "Lattice Boltzmann model for simulating flows with multiple phases and components," Phys. Rev. E 47(3), 1815 (1993).

${ }^{41}$ M. R. Swift, W. R. Osborn, and J. M. Yeomans, "Lattice Boltzmann simulation of nonideal fluids," Phys. Rev. Lett. 75(5), 830 (1995).

${ }^{42}$ M. R. Swift, E. Orlandini, W. R. Osborn, and J. M. Yeomans, "Lattice Boltzmann simulations of liquid-gas and binary fluid systems," Phys. Rev. E 54(5), 5041 (1996).

${ }^{43}$ P. Yuan and L. Schaefer, "Equations of state in a lattice Boltzmann model," Phys. Fluids 18, 042101 (2006).

${ }^{44}$ M. Sbragaglia, R. Benzi, L. Biferale, S. Succi, K. Sugiyama, and F. Toschi, "Generalized lattice Boltzmann method with multirange pseudopotential,” Phys. Rev. E 75(2), 026702 (2007).

${ }^{45}$ A. L. Kupershtokh, D. A. Medvedev, and D. I. Karpov, "On equations of state in a lattice Boltzmann method," Comput. Math. Appl. 58, 965 (2009).

${ }^{46}$ S. V. Lishchuk, C. M. Care, and I. Halliday, "Lattice Boltzmann algorithm for surface tension with greatly reduced microcurrents," Phys. Rev. E 67, 036701 (2003).

${ }^{47}$ M. Latva-Kokko and D. H. Rothman, "Diffusion properties of gradientbased lattice Boltzmann models of immiscible fluids," Phys. Rev. E 71, 056702 (2005).

${ }^{48}$ I. Halliday, R. Law, C. M. Care, and A. Hollis, "Improved simulation of drop dynamics in a shear flow at low Reynolds and capillary number," Phys. Rev. E 73(5), 056708 (2006).

${ }^{49}$ J. U. Brackbill, D. B. Kothe, and C. Zemach, "A continuum method for modeling surface tension,” J. Comput. Phys. 100(2), 335 (1992).

${ }^{50}$ I. Halliday, A. P. Hollis, and C. M. Care, "Lattice Boltzmann algorithm for continuum multicomponent flow,” Phys. Rev. E 76, 026708 (2007).

${ }^{51}$ G. I. Taylor, "The viscosity of a fluid containing small drops of another fluid,” Proc. R. Soc. London, Ser. A 138, 41 (1932).

${ }^{52} \mathrm{Q}$. Zou and X. He, "On pressure and velocity boundary conditions for the lattice Boltzmann BGK model,” Phys. Fluids 9, 1591 (1997).

${ }^{53}$ J. S. Rowlinson and B. Widom, Molecular Theory of Capillarity (Clarendon, London, 1989).

${ }^{54}$ S. Bekri and P. M. Adler, "Dispersion in multiphase flow through porous media,” Int. J. Multiphase Flow 28(4), 665 (2002).

${ }^{55}$ A. Briant, P. Papatzacos, and J. Yeomans, "Lattice Boltzmann simulations of contact line motion in a liquid-gas system," Philos. Trans. R. Soc. London Ser. A 360, 485 (2002).

${ }^{56} \mathrm{~T}$. Lee and L. Liu, "Lattice Boltzmann simulations of micron-scale drop impact on dry surfaces," J. Comput. Phys. 229(20), 8045 (2010).

${ }^{57}$ J. D. Tice, A. D. Lyon, and R. F. Ismagilov, "Effects of viscosity on droplet formation and mixing in microfluidic channels," Anal. Chim. Acta 507, 73 (2004).

${ }^{58}$ J. Tan, S. Li, K. Wang, and G. Luo, "Gas-liquid flow in T-junction microfluidic devices with a new perpendicular rupturing flow route," Chem. Eng. J. 146(3), 428 (2009).

${ }^{59}$ A. Gupta and R. Kumar, "Effect of geometry on droplet formation in the squeezing regime in a microfluidic T-junction," Microfluid. Nanofluid. 8(6), 799 (2010).

${ }^{60}$ S. van der Graaf, M. L. J. Steegmans, R. G. M. van der Sman, C. G. P. H. Schroën, and R. M. Boom, "Droplet formation in a T-shaped microchannel junction: A model system for membrane emulsification," Colloids Surf., A 266, 106 (2005). 